\title{
Coloração Total Absolutamente Equilibrada em uma Família de Grafos Regulares
}

\author{
ANGELO SANTOS SIQUEIRA ${ }^{1}$, ABEL RODOLFO GARCÍA LOZANO $^{2}$, \\ SERGIO RICARDO PEREIRA DE MATTOS $^{3 *}$ e JHOAB NEGREIROS ${ }^{3}$
}

Recebido em 10 de dezembro de 2018 / Aceito em 30 de setembro de 2020

\begin{abstract}
RESUMO. Neste trabalho introduzimos os conceitos de coloração total absolutamente equilibrada e composição de grafos. Provamos que para $n, k \in \mathbb{N}$, se $(k+1) \mid n$, existe um grafo $k$-regular conexo com $n$ vértices que admite uma coloração total absolutamente equilibrada com no máximo $\Delta+2$ cores. Esse resultado mostra que existe uma relação entre a regularidade e o número de vértices do grafo que possibilita a construção de uma família de grafos regulares, denominados grafos harmônicos. Em seguida, mostramos que todo grafo harmônico de grau $k$ pode ser obtido como composição sucessiva de grafos completos de grau $k$. Finalizamos, provando que os grafos harmônicos não possuem vértice de corte, fato que implica que todo grafo desta família possui conectividade de vértices $\kappa(G) \geq 2$.
\end{abstract}

Palavras-chave: grafos harmônicos, composição de grafos, conectividade de vértices.

\section{INTRODUÇÃO}

Em 2011, Friedmann et al [3] mostraram que se um grafo $G(V, E)$ possui uma coloração de vértices com folga de ordem $\Delta$ com $t$ cores, então essa coloração pode ser estendida para uma coloração total de $G$ com no máximo $t+1$ cores. Posteriormente, Lozano et al. [8] provaram que se um grafo regular admite uma coloração com folga de ordem $\Delta$ com $\Delta+1$ cores, então a coloração de vértices pode ser completada para uma coloração total equilibrada com no máximo $\Delta+2$ cores. Em 2016, Lozano, Siqueira e Mattos [5] apresentaram uma heurística, baseada no conceito de famílias consistentes, para coloração total de grafos, que satisfaz a conjectura

\footnotetext{
*Corresponding author: Sergio Ricardo Pereira de Mattos - E-mail: rickdemattos@unigranrio.edu.br

${ }^{1}$ Universidade do Estado do Rio de Janeiro, Departamento de Matemática. Unigranrio, Programa de PósGraduação em Ensino das Ciências, Grupo de Pesquisa em Matemática Discreta e Computacional - E-mail: abel.garcia.lozano@gmail.com https://orcid.org/0000-0002-7607-5928

${ }^{2}$ Unigranrio, Curso de Matemática, Grupo de Pesquisa em Matemática Discreta e Computacional - E-mail: rickdemattos@gmail.com https://orcid.org/0000-0003-2156-4772

${ }^{3}$ Unigranrio, Programa de Pós-Graduação em Humanidades, Culturas e Artes, Grupo de Pesquisa em Matemática Discreta e Computacional - E-mail: asiqueira@unigranrio.edu.br https://orcid.org/0000-0001-6713-4570

${ }^{4}$ Unigranrio, Curso de Matemática, Grupo de Pesquisa em Matemática Discreta e Computacional - E-mail: jhoabnegreiros@gmail.com https://orcid.org/0000-0001-9628-6688
} 
de Vizing-Behzad. O objetivo geral do presente trabalho é apresentar duas formas distintas de se obter famílias de grafos regulares que admitem coloração total absolutamente equilibrada, e apresentar uma importante propriedade desta família de grafos.

Este texto está organizado da seguinte forma: inicialmente revisamos conceitos básicos de grafos e coloração, e apresentamos alguns resultados referentes à coloração de vértice com folga de ordem $\Delta$ que garantem uma extensão natural para coloração total com no máximo $\Delta+2$ cores, que no caso dos grafos regulares é absolutamente equilibrada [3, 8]. Em seguida, mostramos como construir a família dos grafos harmônicos sem utilizar o conceito de produto funcional de grafos [10]. Finalizamos, apresentando um resultado relacionado ao invariante conectividade, mostrando que os grafos harmônicos possuem conectividade de vértices $\kappa(G) \geq 2$.

\section{CONCEITOS BÁSICOS}

Nesta seção, apresentamos definições básicas e terminologias da teoria dos grafos utilizadas no decorrer do trabalho, e que podem ser encontradas em [1,2].

Um grafo simples $G(V, E)$ é uma estrutura composta por um conjunto $V$ de vértices e um conjunto $E$ de arestas, tais que $V$ é finito não vazio e $E$ é formado por subconjuntos de dois elementos de $V$. Representamos, respectivamente, por $|V|$ ou $n$ e por $|E|$ ou $m$, os números de vértices e arestas de um grafo. Dados $u$ e $v$, vértices quaisquer de $G(V, E)$ se existir a aresta $\{u, v\}$, escreveremos $u v$. Se $\{u, v\} \in E(G)$, então dizemos que a aresta é incidente a $u$ e $v$, que $u$ e $v$ são adjacentes, e que $u$ é vizinho de $v$. Para cada vértice $v$, o número de arestas incidentes em $v$ é dito grau do vértice e é representado por $d(v)$. O conjunto de vizinhos de um vértice $v$ de $G$ é denotado por $N_{G}(v)$ ou simplesmente $N(v)$. O número $\Delta(G)=\max \left\{d_{G}(v): v \in V\right\}$ é o grau máximo de $G$. Se todos os vértices de um grafo $G$ tem o mesmo grau $k$, então $G$ é k-regular ou simplesmente regular. Se um grafo $G$ com $n$ vértices é (n-1)-regular, então ele é denominado grafo completo de ordem $\mathbf{n}$ e denotado por $K_{n}$. Um caminho em um grafo $G$ é uma sequência finita e não nula $S=v_{0} e_{1} v_{1} e_{2} \ldots e_{k}, v_{k}$ cujos os termos são alternativamente vértices e arestas, tais que os extremos de $e_{i}$ são $v_{i-1}$ e $v_{i}, \operatorname{com} i=1 \ldots k$, e nenhum elemento de $S$ se repete.

Dados dois grafos $G(V, E)$ e $G^{\prime}\left(V^{\prime}, E^{\prime}\right)$, dizemos que $G^{\prime}$ é subgrafo induzido de $G$, se $V^{\prime} \subset V$ e para todo par de vértices $u, v \in V^{\prime}$ tem-se que $u v \in E^{\prime}$, se e somente se $u v \in E$. Um grafo $G(V, E)$ é dito conexo se para todo par de vértices $x, y \in V$, existe um caminho que liga $x$ e $y$, caso contrário, dizemos que $G$ é desconexo. Se para cada par de vértices $x, y \in V$, existem pelo menos $k$ caminhos disjuntos ligando $x \operatorname{com} y$, então $G(V, E)$ é dito k-conexo. Uma componente conexa de um grafo é qualquer subgrafo induzido conexo. Um vértice $v$ em um grafo conexo é um vértice de corte, se ao removê-lo o grafo torna-se desconexo.

\section{COLORAÇÃO EM GRAFOS}

Nesta seção, apresentamos as definições de coloração de vértices, de arestas, coloração com folga, coloração total e introduzimos o conceito de coloração total absolutamente equilibrada, que servirão de suporte para o restante do trabalho. Estes conceitos, assim como propriedades 
e resultados referentes a essas colorações, podem se encontrados com maior profundidade e detalhamento em $[3,9,11,12]$.

A coloração é um problema clássico em teoria dos grafos. Dado um grafo $G(V, E)$, e um conjunto $C=\left\{c_{1}, c_{2}, c_{3}, \ldots, c_{k}\right\}, k \in \mathbb{N}$, uma coloração de $G$ com os elementos de $C$ é uma aplicação $c: A \subset(V \cup E) \rightarrow C$, tal que dois elementos adjacentes ou incidentes de $A$ possuem sempre imagens distintas. Se $A=V$ é dita coloração de vértices, se $A=E$ é dita coloração de arestas e se $A=(V \cup E)$ é dita coloração total.

Uma coloração de $G(V, E)$ com as cores de $C=\left\{c_{1}, c_{2}, c_{3}, \ldots, c_{k}\right\}, k \in \mathbb{N}$ é equilibrada se, para todo par de cores $c_{i}$ e $c_{j}$, tal que $i \neq j$, tem-se $\left|a\left(c_{i}\right)-a\left(c_{j}\right)\right| \leq 1$ com $i, j=1, \ldots k$ e $a\left(c_{i}\right)$ e $a\left(c_{j}\right)$ representam, respectivamente, os números de aparições das cores $c_{i}$ e $c_{j}$ na coloração.

Definição 3.1. Dado um grafo $G(V, E)$, um conjunto de cores $C=\left\{c_{1}, c_{2}, c_{3}, \ldots, c_{k}\right\}, k \in \mathbb{N} e$ uma coloração c $: S \subseteq(E \cup V) \rightarrow C$ de $G$. A coloração c é absolutamente equilibrada se, para todo par de cores tal que $i \neq j$, tem-se $a\left(c_{i}\right)=a\left(c_{j}\right)$.

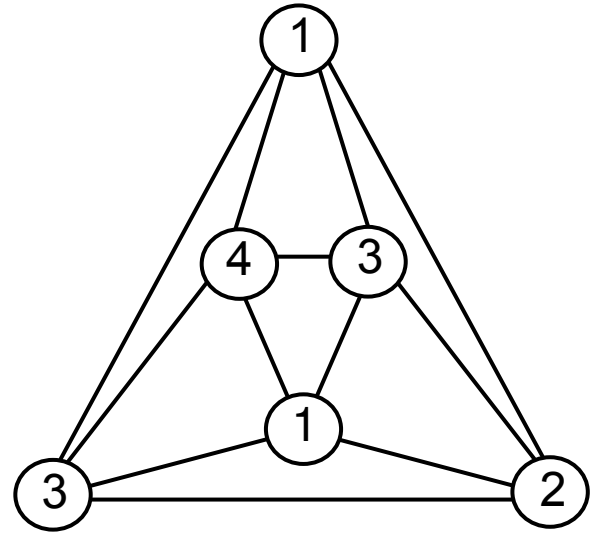

(a) Coloração Equilibrada

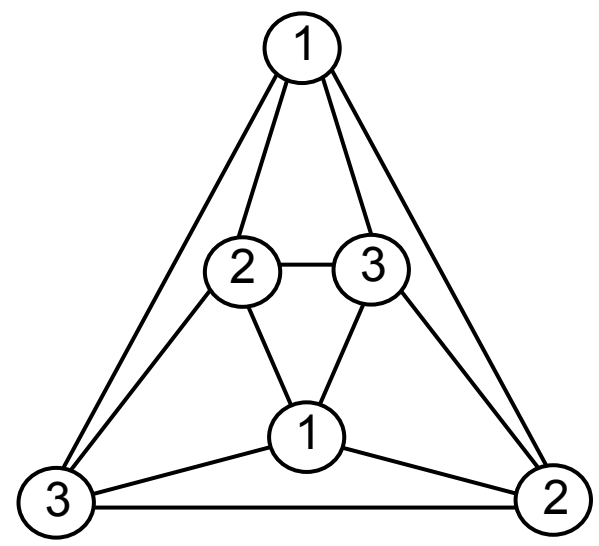

(b) Coloração Absolutamente Equilibrada

Figura 1

Definição 3.2. Seja um grafo $G(V, E)$ e um conjunto de cores $C=\left\{c_{1}, c_{2}, c_{3}, \ldots, c_{p}\right\}$ com $p \in \mathbb{N}$, seja ainda $|c(N(v))|$ a cardinalidade do conjunto de cores da vizinhança de $v$, uma aplicação $f: V \rightarrow C$ é uma coloração de vértices com folga de ordem $\boldsymbol{k}$ de $G$ se para todo $v \in V$ :

- se $d(v)<k$, então $|c(N(v))|=d(v)$;

- se $d(v) \geq k$, então $|c(N(v))| \geq k$.

Observe que na coloração de vértices com folga de ordem $k$, os vértices com grau menor que a $k$ devem ter todos os vizinhos coloridos com cores distintas; já os de grau igual ou maior do que $k$ 


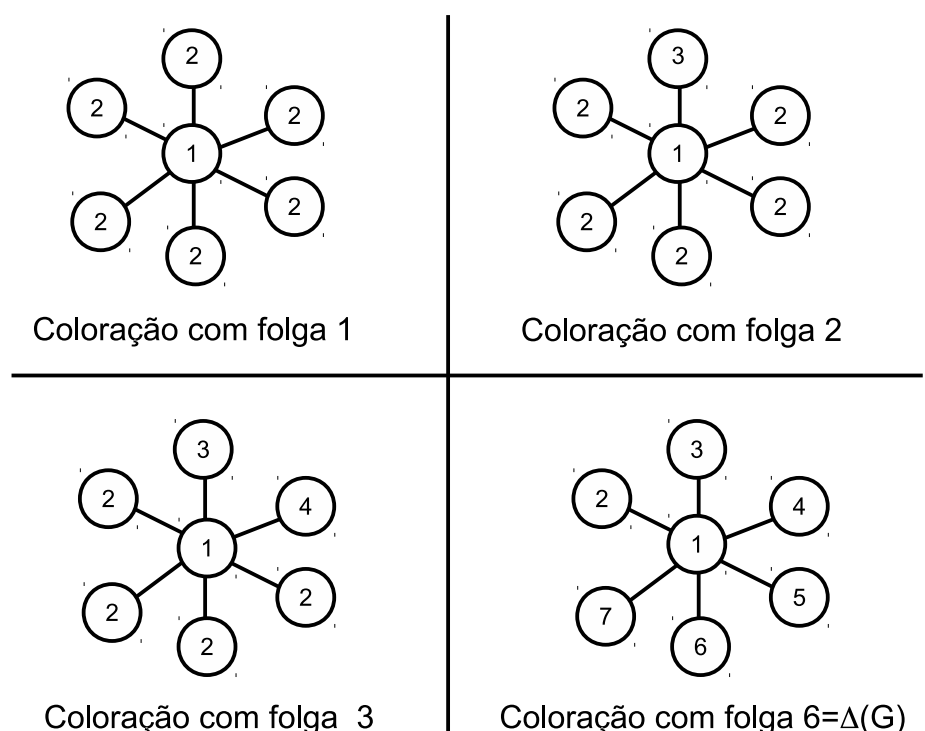

Coloração com folga 3

Coloração com folga $6=\Delta(G)$

Figura 2: Coloração com folga de ordem 1, 2, 3 e $6=\Delta(G)$, respectivamente.

devem utilizar pelo menos $k$ cores na coloração de sua vizinhança. Para $k=1$, temos a coloração usual de vértices e para $k=\Delta$ temos a coloração 2-distante [9].

\section{RELAÇÃO ENTRE COLORAÇÃO COM FOLGA E COLORAÇÃO TOTAL ABSOLUTAMENTE EQUILIBRADA EM GRAFOS REGULARES}

Nesta seção, apresentamos três teoremas que têm como objetivo mostrar que se um grafo regular pode ser colorido com folga $\Delta$ com $\Delta+1$ cores, então existe uma extensão da coloração com no máximo $\Delta+2$ cores e essa extensão é absolutamente equilibrada. Esses resultados podem ser encontrados em $[3,8]$.

Teorema 4.1. [8] Sejam $G(V, E)$ um grafo regular e $c: V \rightarrow C=\{1,2, \ldots \Delta+1\}$ uma coloração com folga de ordem $\Delta$. Então:

(a) $\Delta+1$ divide $|V|$;

(b) Cada cor $i \in C$, é usada exatamente $\frac{|V|}{\Delta+1}$ vezes.

Teorema 4.2. [3] Sejam $G(V, E)$ um grafo com grau máximo $\Delta$ e $c: V \rightarrow C=\{1,2,3, \ldots k\}$, uma coloração com folga de ordem $\Delta$ de G. Então, existe uma coloração total de $G$ com no máximo $k+1$ cores. 
Teorema 4.3. [8] Sejam $G(V, E)$ um grafo regular e $c: V \rightarrow C=\{1,2, \ldots \Delta+1\}$ uma coloração com folga de ordem $\Delta$. Então, existe uma coloração total absolutamente equilibrada de $G$ com no máximo $\Delta+2$ cores.

\section{GRAFOS HARMÔNICOS}

Em 2013, Lozano et al. [4] introduziram o conceito de produto funcional de grafos e de grafos $k$-suporte para auxiliar na costrução dos grafos harmônicos. Mais tarde, Lozano et al. [6] mostraram que o produto funcional permite gerar infinitos grafos harmônicos, a partir de qualquer grafo regular. Nesta seção, apresentamos uma forma de se obter os grafos harmônicos sem utilizar o produto funcional de grafos [10], mostramos que existe uma relação entre a regularidade e número de vértices do grafo que possibilita a construção dos grafos harmônicos. Em seguida, provamos que todo grafo harmônico de grau $k$ pode ser obtido como composição sucessiva de grafos completos de grau $k$. Finalizamos, provando que os grafos harmônicos não possuem vértices de corte.

Definição 5.3. [8] Um grafo $G(V, E)$ regular é dito harmônico, se admite uma coloração de vértices com folga $\Delta$ com $\Delta+1$ cores.

Teorema 5.4. [10] Sejam $n$ e $k \in \mathbb{N}$, se $(k+1) \mid n$, então existe um grafo conexo harmônico $k$-regular com $n$ vértices.

Proof. Sejam $n, k$ e $t \in \mathbb{N}$ tais que $n=t \cdot(k+1)$, se $t=1$, então o grafo $K_{n}$ é o grafo procurado. Caso contrário, vamos construir o grafo $G(V, E)$ como se segue:

Seja $V=\left\{v_{10}, \ldots, v_{1 k}, v_{20}, \ldots, v_{2 k}, \ldots, v_{t 0}, \ldots, v_{t k}\right\}$. Definimos o conjunto $E$ de arestas da seguinte forma:

Dois vértices $v_{i j}$ e $v_{i^{\prime} j^{\prime}}, \operatorname{com} i, i^{\prime} \in\{1,2, \ldots, t\}$ e $j, j^{\prime} \in\{0,1,2, \ldots, k\}$, são adjacentes quando:

- Para $i=i^{\prime}$, se $j=0$, então $j^{\prime} \neq 1$;

- Para $i^{\prime}=i+1(\bmod t)$, se $j=0$, então $j^{\prime}=1$.

A cada vértice $v_{i j}$, damos a cor $j$ do conjunto $\{0,1,2, \ldots, k\}$. O resultado é uma coloração com folga $\Delta(G)$ que utiliza $\Delta+1$ cores. Logo, o grafo $G$ é harmônico.

As figuras 3, 4, 5 e 6 ilustram a prova do Teorema 5.4. Para exemplificar, adotamos $n=15, k=4$ e $t=3$.

Observe que todo grafo harmônico, por definição, satisfaz o Teorema 4.1. Nesse sentido, o resultado oferece uma condição necessária para existência dessa família de grafos regulares. $\mathrm{Na}$ sequência, introduzimos o conceito de grafo composto e apresentamos um resultado que mostra que todo grafo harmônico de grau $k$ pode ser obtido como composição sucessiva de grafos completos de grau $k$. 
(v)

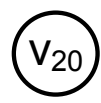

(

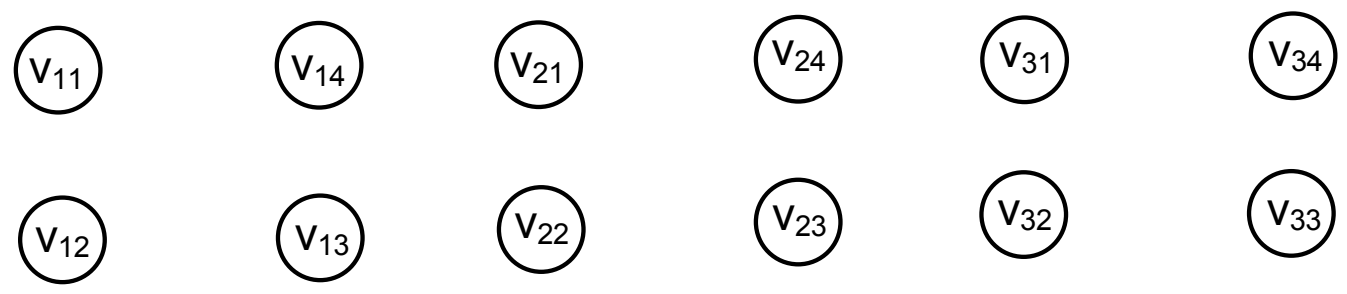

Figura 3: Conjunto de Vértices $V=\left\{v_{10}, \ldots, v_{14}, v_{20}, \ldots, v_{24}, \ldots, v_{t 0}, \ldots, v_{34}\right\}$.
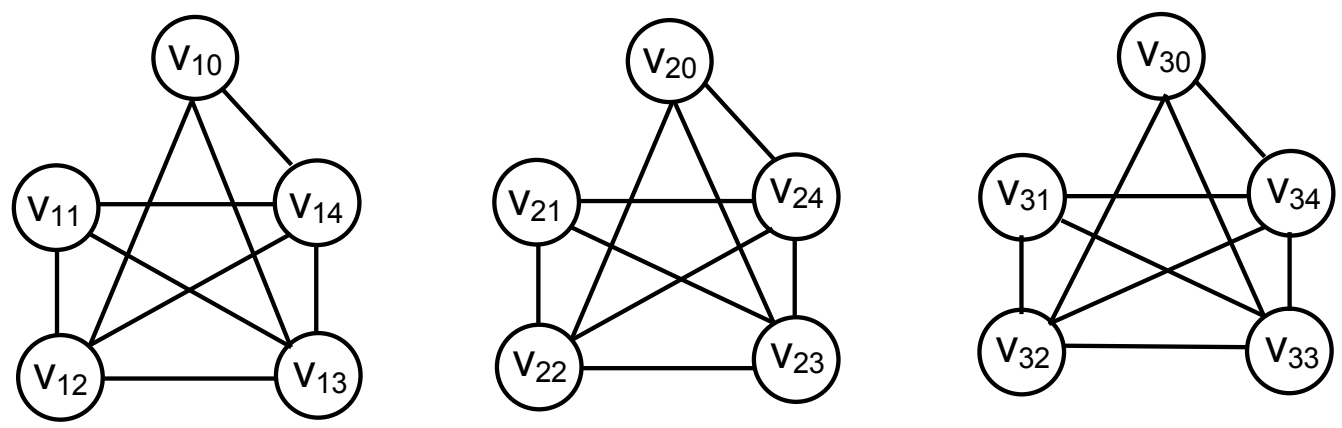

Figura 4: Conjunto de arestas de $G(V, E)$, de acordo com a primeira condição.

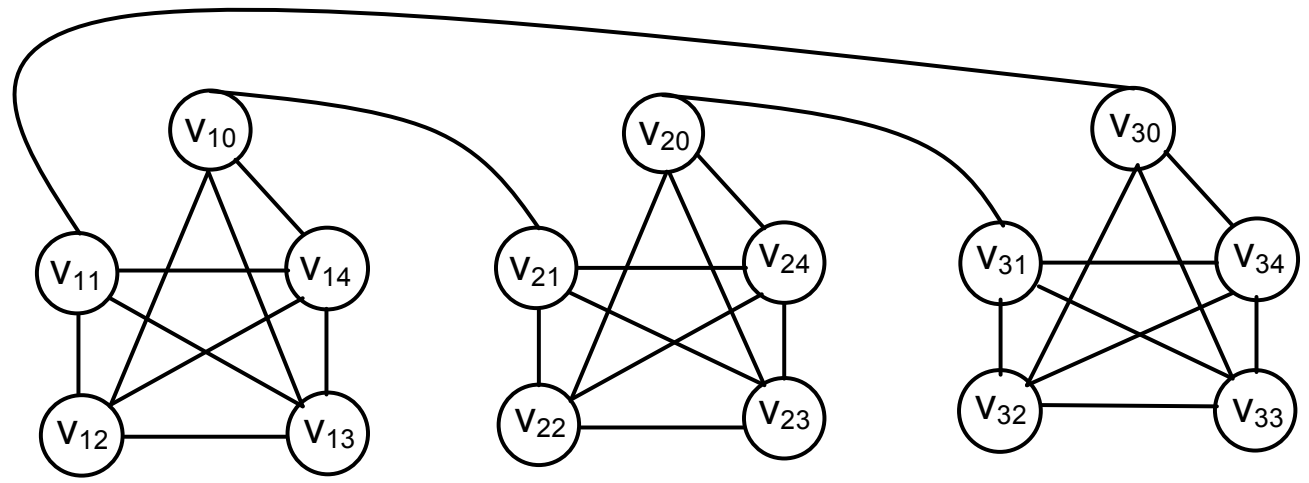

Figura 5: Conjunto de arestas de $G(V, E)$, de acordo com a primeira e a segunda condições. 


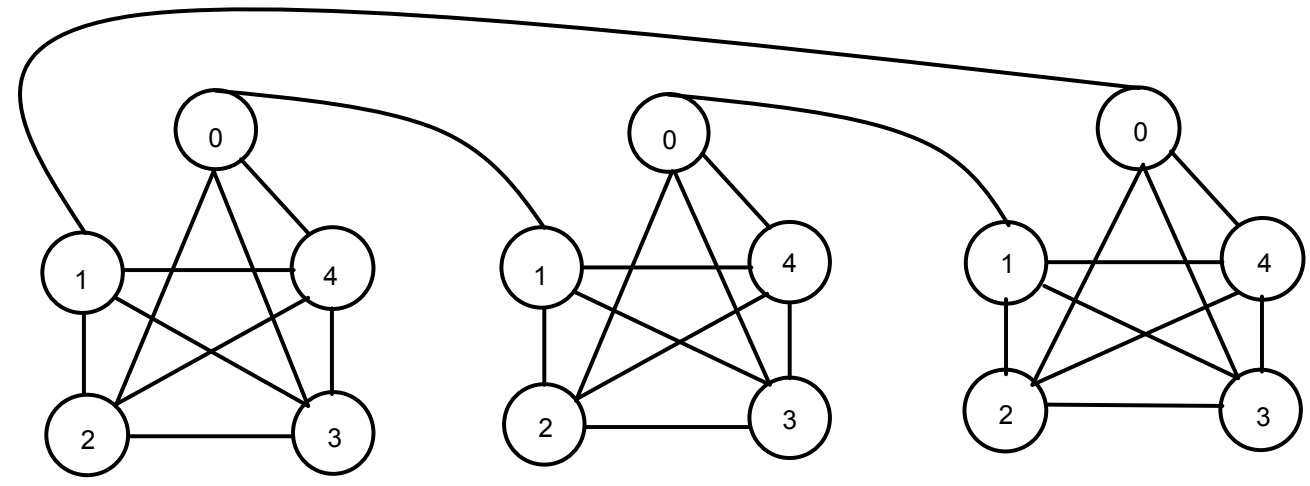

Figura 6: Grafo harmônico (4-regular) colorido com folga 4 com 5 cores.

Definição 5.4. Dados dois grafos harmônicos k-regulares $G_{1}\left(V_{1}, E_{1}\right)$ e $G_{2}\left(V_{2}, E_{2}\right)$, duas colorações com folga $c_{1}: V_{1} \rightarrow C=\{0,1,2, \ldots \Delta\}$ e $c_{2}: V_{2} \rightarrow C=\{0,1,2, \ldots \Delta\}$ e dois subconjuntos de arestas $S_{1}=\left\{u_{1} v_{1}, \ldots u_{k} v_{k}\right\} \subset E_{1}$ e $S_{2}=\left\{x_{1} y_{1}, \ldots x_{k} y_{k}\right\} \subset E_{2}$, tais que $c_{1}\left(u_{i}\right)=c_{2}(x i)$ e $c_{1}\left(v_{i}\right)=c_{2}(y i)$. O grafo $G(V, E)$, tal que $V=\left(V_{1} \cup V_{2}\right)$ e ab $\in E$ se, somente se, uma das seguintes afirmações for verdadeira:

1. $a b \in E_{1}$ e $a b \notin E_{2}$ ou $a b \in E_{2}$ e $a b \notin E_{1}$;

2. $a=u_{i}$ e $b=y_{i}$ ou $a=x_{i}$ e $b=v_{i}$ para algum $i \in\{1,2, \ldots k\}$.

é chamado grafo composto por $G_{1}\left(V_{1}, E_{1}\right)$ e $G_{2}\left(V_{2}, E_{2}\right)$.

Teorema 5.5. Para todo grafo harmônico $G(V, E)$ não completo, existem dois grafos harmônicos $G_{1}\left(V_{1}, E_{1}\right)$ e $G_{2}\left(V_{2}, E_{2}\right)$ (não necessariamente conexos), tais que $G$ é composto por $G_{1} e G_{2}$.

Proof. Seja $c: V \rightarrow C=\{0,1,2, \ldots \Delta\}$ uma coloração de $G$ com folga $\Delta$. Sejam $V_{1}=$ $\left\{v_{0}, v_{1}, \ldots v_{\Delta}\right\}$ um subconjunto de vértices, tal que $c\left(v_{i}\right) \neq c(v j)$ para todo $i, j \in C=$ $\{0,1,2, \ldots \Delta\}$. Sem perda de generalidade, podemos supor que $c\left(v_{i}\right)=i ; i=\{0,1,2, \ldots \Delta\}$. Seja $G_{1}\left(V_{1}, E_{1}\right)$ o grafo induzido por $V_{1}$ e seja $G_{2}\left(V_{2}, E_{2}\right)$ o grafo induzido por $V-V_{1}$. Escolhemos um par de vértices $v_{i}, v_{j} \in V_{1}$, tal que $v_{i} v_{j} \notin E_{1}$. Como o grafo $G(V, E)$ é harmônico, existe um emparelhamento que satura os vértices com as cores $i, j \in C$ e existem vértices $x, y \in E_{2}$, tais que $c(x)=j, c(y)=i$ e $v_{i} x \in E, v_{j} y \in E$. Agora incorporamos uma aresta em $E_{1}$ e outra em $E_{2}$ da seguinte forma: $E_{1} \rightarrow E_{1} \cup\left\{v_{i} v_{j}\right\}$ e $E_{2} \rightarrow E_{2} \cup\{x y\}$. Repetimos esse processo até que $G_{1}$ seja um grafo completo. Logo, por construção $G_{1}$ e $G_{2}$ são os grafos procurados.

Do Teorema 5.5 se obtém, de forma imediata, o seguinte colorário.

Corolário 5.5.1. Todo grafo harmônico de grau $k$ pode ser obtido como composição sucessiva de grafos completos de grau $k$. 
Vale destacar que os grafos harmônicos apresentam propriedades relacionadas ao invariante conectividade que favorecem sua aplicação em sistemas computacionais multiagentes [7]. $\mathrm{O}$ próximo Teorema prova que os grafos harmônicos não possuem vértices de corte, tem como consequência que todo grafo harmônico possui conectividade de vértices $\kappa(G) \geq 2$.

Teorema 5.6. Os grafos harmônicos não possuem vértices de corte.

Proof. Sejam $G(V, E)$ um grafo harmônico e $c: V \rightarrow C=\{0,1,2, \ldots, \Delta\}$ uma coloração com folga $\Delta$ dos vértices de $G$. Suponhamos por absurdo que $G$ possui um vértice de corte $u \in V$ e sem perder a generalidade suponhamos que o vértice $u$ foi colorido com a cor 0 . Seja $G^{\prime}\left(V^{\prime}, E^{\prime}\right)$ uma das componentes conexas obtidas ao se retirar $u$ do grafo $G$. Observe que as cores de $C-\{0\}=$ $\{1,2, \ldots, \Delta\}$ são usadas o mesmo número de vezes em $G^{\prime}$, pois em uma coloração com folga $\Delta$ todos os vértices adjacentes são coloridos com cores distintas, logo dadas duas cores arbitrárias $i \in C-\{0\}$ e $j \in C-\{0\}$ todo vértice de $V^{\prime}$ com a cor $i$ possui um e somente um vizinho com a cor $j$. Denotemos por $V_{i}^{\prime}$ o conjunto de vértices de $G^{\prime}$ coloridos com a cor $i \in C$, seja $q=\left|V_{i}\right|, i \in C-\{0\}$ se $\left|V_{0}^{\prime}\right|=q$, então todos os vértices de $V^{\prime}$ coloridos com cor diferente de 0 têm um vizinho em $V_{0}^{\prime}$, logo nenhum deles pode ser vizinho de $u$, o que é um absurdo. Se $\left|V_{0}^{\prime}\right|<q$, então existem pelo menos $\Delta$ vértices de $G^{\prime}$ com cor diferente de 0 que não possuem vizinho em $V_{0}^{\prime}$. Mas o número de vizinhos de $u$ em $G^{\prime}$ é menor que $\Delta$, logo existem vértices de $V^{\prime}$ com cor diferente de 0 que não tem vizinho com a cor 0 , o que é um absurdo.

Desse resultado se obtém o seguinte colorário.

Corolário 5.6.2. Sejam $u, v \in V$ dois vértices quaisquer de $G(V, E)$, se $G$ é um grafo harmônico, então existe um ciclo em $G$ que contém u e v.

\section{CONCLUSÕES}

Neste trabalho, introduzimos os conceitos de coloração total absolutamente equilibrada e composição de grafos. Mostramos que existe uma relação entre a regularidade e o número de vértices do grafo que possibilita a construção de uma família de grafos regulares, denominada grafos harmônicos. Provamos que todo grafo harmônico de grau $k$ pode ser obtido como composição sucessiva de grafos completos de grau $k$. Por fim, mostramos que os grafos harmônicos não possuem vértice de corte e obtemos como consequência desse resultado o fato de que todo grafo harmônico possui conectividade de vértices $\kappa(G) \geq 2$.

\footnotetext{
ABSTRACT. In this work we introduce the concepts of absolute equitable total coloring and graph composition. We prove that for $n, k \in \mathbb{N}$, if $(k+1) \mid n$, there is a connected $k-$ regular graph with $n$ vertices that admits a absolute equitable total coloring, with at most Delta +2 colors. This result shows that there is a relationship between regularity and the number of vertices of the graph that makes it possible to build a family of regular graphs, called harmonic graphs. Then, we show that every harmonic graph of degree $k$ can be obtained as successive composition of complete graphs of degree $k$. We conclude by proving that
} 
the harmonic graphs do not have a cut vertex, that implies that every graph of this family has vertex connectivity $\kappa(G) \geq 2$.

Keywords: harmonic graphs, graph composition, vertex connectivity.

\section{REFERÊNCIAS}

[1] J. Bondy \& U. Murty. "Graph Theory with Applications”. North-Holland, New York (1976).

[2] R. Diestel. "Graph Theory”. Springer-Verlag, New York (1997).

[3] C. V. P. Friedmann, A. R. G. Lozano, L. Markenzon \& C. F. E. M. Waga. Total coloring of Blockcactus graphs. The journal of combinatorial mathematics and combinatorial computing, 78 (2011) $273-283$.

[4] A. R. G. Lozano, A. S. Siqueira, S. Jurkiewicz \& C.V.P. Friedmann. Produto Funcional de Grafos. TEMA, 14 (2013), 221-232.

[5] A. R. G. Lozano, A. S. Siqueira \& S. R. P. Mattos. Famílias Consistentes e a Coloração Total de Grafos. Proceeding Series of the Brazilian Society of Computational and Applied Mathematics, volume 5 (2017). DOI: 10.5540/03.2017.005.01.0230.

[6] A. R. G. Lozano, A. S. Siqueira, S. R. P. Mattos \& S. Jurkiewicz. Produto funcional de grafos: um modelo para conexão de sistemas multiagentes. TEMA, 17 (2016), 341-352.

[7] A. R. G. Lozano, A. S. Siqueira \& S. R. P. Mattos. Functional Product of Graphs and Multiagent Systems. Global Journal of Science Frontier Research: Mathematics and Decision Sciences, 17 (2017), $15-28$.

[8] A. R. G. Lozano, A. S. Siqueira, C. V. P. Friedmann \& S. Jurkiewicz. Relationship between equitable total coloring and range coloring in some regular graphs. Pesquisa Operacional, 28 (2016), 161-171.

[9] A. R. G. Lozano, C. V. P. Friedmann, C. F. E. M. Waga \& L. Markenzon. Coloração de Vértices com Folga. Anais do Simpósio Brasileiro de Pesquisa Operacional (XLI SBPO), Porto Seguro, Bahia, Brasil (2009).

[10] S. R. P. Mattos. "Produto Funcional de Grafos: Propriedades e Aplicações". Tese de Doutorado. COPPE, UFRJ, Rio de Janeiro, RJ, (2017).

[11] A. S. Siqueira. "Coloração Total Equilibrada em Subfamílias de Grafos Regulares". Tese de Doutorado. COPPE, UFRJ, Rio de Janeiro, RJ (2011).

[12] H. Yap. "Total colourings of graphs". Springer, Berlin (1996). 